\title{
Legal Responsibility on Errors of the Artificial Intelligence- based Robots
}

\author{
Viony Kresna Sumantri \\ Tarumanagara University, Indonesia \\ vio.kresna@gmail.com
}

\begin{abstract}
Modern technology is developing rapidly. One branch of industrial technology that is particularly popular at the moment is artificial intelligence (AI) that facilitates society's daily life. On smartphones, artificial intelligence can be found in map applications, personal assistants, shopping websites, and various other applications. Saudi Arabia granted an AI-based robot named Sophia citizenship, and the Shibuya Mirai robot was granted a residence permit by Japan. AI-based technology is used every day and has become a common thing in various parts of the world; however, in Indonesia, legal regulations regarding AI do not yet exist. As a result, a legal vacuum has emerged. When a loss occurs, responsibility can be borne by various parties ranging from consumers, producers, third parties (such as robot trainers or shipping couriers) to the robot itself. Which party will be determined responsible depends upon how a country positions AI. If Indonesia follows in Saudi Arabia's footsteps, then the responsibility will be borne by the AI robot as a citizen. The robot will have the right to sue and be sued, to get the same position before the law, including other rights and obligations, enjoyed by human citizens. Artificial intelligence law-making is a very complicated process and will involve many parties. How Indonesia positions AI is very crucial, particularly in the event of harm or danger caused by AI systems. Various frameworks and concepts can be used, ranging from equating artificial intelligence to living beings, such as humans, pets, or ordinary products to creating entirely new concepts for a legal framework regulating AI-based systems.
\end{abstract}

KEYWORDS: Artificial Intelligence, Responsibility, AI Law.

Copyright $\odot 2019$ by Author(s)

This work is licensed under a Creative Commons Attribution-ShareAlike 4.0 International License. All writings published in this journal are personal views of the authors and do not represent the views of this journal and the author's affiliated institutions.

\section{HOW TO CITE:}

Sumantri, Viony Kresna. "Legal Responsibility on Errors of the Artificial Intelligence-based Robots" (2019) 6:2 Lentera Hukum 337-352.

Submitted: April 03, 2019 Revised: June 04, 2019 Accepted: July 31, 2019 


\section{INTRODUCTION}

The global technology industry is developing very fast. Nowadays, humans have been able to realize ideas that were previously considered imagination only found in novels and films. Until now, smartphones, digital map applications, autonomous cars, and robots could only found on film. Another previously fantastical contemporary technological development is artificial intelligence (AI). AI is the theory and development of computer systems that can complete tasks which typically require human intelligence, such as visual perception, speech recognition, decision-making, and language translation. ${ }^{1}$ Despite its futuristic nature, AI is not new; the concept of AI existed several decades ago, as early as 1950. AI was first introduced by Alan Turing, a computer scientist from England when he was breaking the Enigma code used by Germany to send secret messages during World War II.

At that time, Turing and his teammates managed to make a machine that could predict and break the code. Their machine saved tens of millions of lives and shortened the war. Turing's machine became the basis of current AI technology. Although the phrase "artificial intelligence" was not used at the time, it was later on introduced by American computer scientist John McCarthy while discussing the ability of machines to think for themselves at the 1956 Dartmouth Conference. When humans communicate with others through language, they need almost no effort. This process is a complex and challenging thing to understand. It is difficult to develop a computer system that is able to make and understand the language used by humans. ${ }^{2}$ Most AI discoveries during the 1960s were the result of research on problem-solving techniques applied to computer systems so that they could solve simple puzzles, play games, and retrieve information. ${ }^{3}$

In 1961, the first industrial robot was created to replace human work. Such a robot was intended to replace hazardous work typically done by humans. In 1980, Japan's Waseda University created a robot named WABOT, who can play the piano. WABOT can also talk to humans, read music scores, and accompany singers. WABOT became a model for making personal robots. In 1986, European car manufacturer Mercedes Benz made the first driverless car, equipped with cameras and sensors.

For the first time, humanity lives in an era where there is an intelligence greater than humans: AI. ${ }^{4}$ AI is literally in human hands as smartphone personal assistants, such as Google Assistant on Android-based smartphones and Siri on Apple-based smartphones. Amazon's Alexa and the Google Home personal assistant serve similar functions. These personal assistants are designed to do various jobs that facilitate human life, including playing music, opening desired applications through voice recognition technology, making an agenda, making reservations at restaurants, sending and reading messages, purchasing various items and taking notes as needed. Siri has

\footnotetext{
The New Oxford American Dictionary, Third Edition.

Nils J. Nilson, "Principles of Artificial Intelligence", San Francisco, Elsevier Science at 13.

Ibid at 32.

James Barrat, "Artificial Intelligence and The End of The Human Era: Our Final Invention," New York, Macmillan Publishers at 14.
} 
been around since the end of 2011, while Google Assistant was only launched three years ago, in 2016. Although it was not as popular, long before Google Assistant and Siri, there was Eliza. Eliza was created in 1964 by the Massachusetts Institute of Technology (MIT) Artificial Intelligence Laboratory and is the first computer program capable of having conversations with humans. Unfortunately, Eliza failed to pass the Turing test, but the discovery of Eliza became the basis of making the chatbot (conversational robot).

Although the positive impact of AI on human life is overwhelming, it cannot be denied that AI has also carried out a variety of adverse and undesirable actions on behalf of humans. For example, in 2015 in Switzerland, several artists assigned robots to buy goods randomly from an online store and exhibited the results in the gallery of St. Gallen's Kunst Halle. The robot's unregulated purchases included ecstasy pills, fake passports, shoes, and a few other items. Police made arrests of robots, but the robot makers were not arrested, and the robot was later released after police confiscated ecstasy pills and other evidence. Similarly, in 2016, Microsoft launched a conversational robot named Tay that operates on the social network Twitter. Tay studies and retrieves data from human conversations on Twitter, and the data learns to communicate. At first Tay produced a normal conversation; however, after only 16 hours of operation, Tay was deactivated because he made several conversations that offended SARA (Tribe, Religion, Race, and Intergroup).

In 2017, an autonomous car belonging to the Uber company crashed into a pedestrian who was crossing the road, causing the pedestrian's death. There was a driver in the car at the time; however, the car was in self-driving mode and was therefore not directly operated by humans. In the end, the victim's family agreed to settle the problem outside the court. Of the various incidents that have occurred, we can conclude that there is no equivalent law enforcement for AI. If the above activities are carried out by humans themselves, inevitably they would face punishment according to extant provisions in force in each country. Even in some criminal cases, if someone commits a crime and is not legally reprimanded, public demonstrations demand justice and punishment. Still, when the AI mentioned above incidents occurred, the public did not react. Enforcement and justice remain the responsibility of law enforcement.

It turns out that the absence of law enforcement for AI crimes is due to a legal vacuum surrounding AI itself. Until now, no country has made legal arrangements concerning AI, even though AI can, in fact, be quite dangerous for humans. Many AI experts have expressed concern about the development and use of AI technology. For example, Kenneth Stanley, an AI expert employed at Uber Artificial Intelligence Labs, expressed concern over AI being used to hurt humans. Irakli Beridze, chair of Artificial Intelligence and Robotics at the United Nations Interregional Crime and Justice Research Institute (UNICRI), similarly expressed concern about the development of AI, arguing that it could be used by criminals and terrorist organizations to harm 
humans. ${ }^{5}$ In general, responsibility for errors can be borne by product consumers and manufacturers. However, after Saudi Arabia acknowledged the Sophia robot as a citizen, it did not rule out responsibility placed on the robot itself. Contrastingly, the European Commission proposed that AI-based robots be given the right to be prosecuted like humans. Burkhard Schafer, a professor at the University of Edinburgh, likens the responsibilities of AI ownership to those of pet ownership. ${ }^{6}$

\section{ROBOT-BASED ARTIFICIAL INTELLIGENCE}

$\mathrm{AI}$ is one of the fields of computer science that focuses on making artificial intelligence that can work and react just like human intelligence. ${ }^{7}$ AI is closely related to research in the human brain. Researchers believe that AI can be perfected by understanding how the human brain works. By imitating the way the human brain learns, thinks, and acts, researchers can create machines that do the same thing. ${ }^{8}$ Put, AI imitates by gathering information or data, analyzing it, and then providing a result or reaction to that information. For example, in self-driving car technology, AI systems must complete the human task of going somewhere. If AI examines its surrounding conditions and is unable to operate safely, the AI system can also ask humans to take control of their vehicles. Of course, by making this AI, many have come to expect that future AI will be able to replace a variety of jobs requiring human intelligence.

The main difference between an ordinary machine with a robot and robot-based AI is the ability to learn and make decisions. Consider, for example, ordinary robots, such as toaster ovens that are used in everyday life. Humans can initiate orders by pressing the button found on the toaster oven. Then, the machine will operate according to the command. Humans must determine the appropriate temperature and duration of time to ensure their food will cook perfectly. Contrastingly, AI-based robots can detect and determine minute details, such as the temperature and time required. However, when AI operates, it does not rule out the possibility of various undesirable incidents, which could even be considered detrimental to humans. For example, in autonomous car technology, AI could potentially identify surrounding objects incorrectly or too late, incur AI system damage, or experience several other factors making it unable to fulfill its work. Misidentification can be the result of pure AI errors as well as non-AI problems, such as damage to the camera. Whenever any loss occurs, a new problem arises: who should be held responsible when AI makes mistakes that harm humans?

5 Dan Robitzski, "Five Experts Share What Scares Them the Most About AI", Futurism, https://futurism.com/artificial-intelligence-experts-fear, accessed on March $23^{\text {rd }}, 2019$.

6 Rose Eleveth, "My Robot Bought Illegal Drugs”, BBC,http://www.bbc.com/future/story/20150721-myrobot-bought-illegal-drugs, accessed on March $18^{\text {th }}, 2019$.

7 Techopedia, "Artificial Intelligence (AI)", Techopedia, https:/www.techopedia.com/definition /190/artificial-intelligence-ai, accessed on March 26 ${ }^{\text {th }}, 2019$.

8 Prateek Joshi, "Artificial Intelligence with Phyton", Birmingham, Packt Publishing at 7. 
In 1979, a factory worker named Robert Williams, who was working in a Ford automobile manufacturing plant was killed by a robot. When he was moving goods to a storage room, a robot's hand slammed him to death. This happened because the robot misidentified Williams as an object to be placed in the storage room. The Williams case is considered the first human death caused by a robot. The Ford Company then provided compensation of USD 10,000,000 to the family of the victim. Again, if death is caused by humans, USD 10,000,000 is undoubtedly not considered enough to replace someone's life. Until now, there have been various incidents of harm caused by robots, from small losses to death, but no punitive measures are applied in addition to monetary compensation.

AI technology received particular attention in 2017 when a robot created by Hanson's Robotics named Sophia was made a citizen of Saudi Arabia. This is, of course, a huge step taken by Saudi Arabia; however, if observed and reviewed mainly from a legal perspective, at the time Sophia did not meet the qualifications to become a citizen of Saudi Arabia. According to the laws in force, citizenship can be obtained through birth, marriage, and naturalization. Obtaining citizenship through naturalization must meet specific requirements. Candidates for naturalization must have reached a sufficient age; be considered clinically sane; live in-country for ten consecutive with permanent residence (PR) for five years; have an income; have no criminal record; and be able to read, write, and speak Arabic fluently. Despite not fulfilling a number of these requirements, which must be fulfilled by humans, Sophia was still recognized as a citizen of Saudi Arabia. Sophia's citizenship affects not only the legal framework of citizenship for Saudia Arabia, but also its culture. Appointing robots as citizens can produce a new culture or even contradict or challenge a country's existing sociocultural values.

In Japan, a robot named Shibuya Mirai became the first conversational robot granted a residence permit. In order to obtain a residential permit in Japan, there are likewise several conditions that must be met. One must have lived in Japan for ten years, or three years for those who are married to a Japanese citizen. Second, a person never committed a crime; and made contributions to Japan. In addition, when Shibuya Mirai was made, he was only seven years old. Although granted a residence permit, Shibuya Mirai does not actually have a physicality, but rather is a software or system in the Line conversation app. Just like Sophia, Shibuya Mirai does not meet the majority of existing citizenship requirements; however, Shibuya Mirai is considered to satisfy the third criterion, insofar as he has already contributed to Japan. Shibuya Mirai is undoubtedly a new national icon as a leading and innovative technology which takes the form of a cheerful boy with a photography hobby. Still, faced with the same criteria and the same characteristics, humans with an identical citizenship application would most certainly be rejected. Furthermore, neither Sophia nor Shibuya Mirai actually applied for their citizenship or residence permits.

The appointment of Sophia and Shibuya Mirai can also be an example for all countries interested in legally recognizing AI. The presence of AI technology will be 
more and more common in human life, both in the form of applications and robots that resemble humans. Granting citizenship and residence permits can be seen as a country's show of appreciation and support for the advancement of AI technology. Nevertheless, AI citizenship is clearly confusing international society, especially when the consequences of robot citizenship remain unclear. Do robots such as Sophia and Shibuya Mirai have the same rights and obligations as other humans, or is appointment only a way for their host countries to be superior to other countries? Various questions arise, ranging from the robot's rights and obligations to how the robot is adapted to national culture. In Saudi Arabia, a woman must be accompanied by a man from her family when leaving the house. Such a law raises questions when applied to the Sophia robot, which is made to resemble a woman and uses a female voice. Does the same law apply to Sophia? Also questioned is the right of Sophia to participate in general elections, among many other activities considered citizenship rights. Unfortunately, at the time of receiving citizenship, Saudi Arabia did not explain the Sophia robot's rights and obligations.

Elon Musk, CEO of Tesla and SpaceX has expressed concern over the dangers of AI-based technology. Elon Musk argued that AI technology could be more dangerous than nuclear technology concerning human extinction. He added that developed countries could weaponize computer science and AI to wage a third World War. He emphasized the importance of AI regulations, arguing that legal frameworks are needed before more complicated AI incidents arise and waiting for the development of better, less hazardous AI will only result in regulations being too little, too late.

AI experts have their concerns about AI development, yet all of these concerns are related to the impact of AI on human security and its potentially harmful impact on humans. Many AI experts even urged the government to make AI regulations immediately. Surely concern even by AI experts is not a positive sign for a potentially unregulated future.

Today, only basic principles govern AI, namely the Asilomar Artificial Intelligence Principles created at the 2017 Asilomar Conference. In total, there are 23 Asilomar Principles: (1) Research objectives of AI must have directional benefits; (2) Research funding and investment in AI must be accompanied by funding for research that can ensure the benefits of using AI in computer science, economics, law, ethics and social aspects in human life; (3) There must be a constructive and healthy exchange between AI researchers and lawmakers; (4) Researchers and AI developers must have a cooperative and transparent culture; (5) AI developers must be cooperative and not eliminate existing safety standards; (6) The entire AI system must have guaranteeable safety during the period of its use; (7) If the AI system causes a loss, the cause of this loss must be known; (8) A court's decision to utilize an AI system must be followed by an explanation that can be audited by the competent (human) authority; (9) The designers and producers of AI systems are responsible for moral damage from AI users; (10) AI systems must be designed so that all operation is in accordance with the goals and behaviour of humanity; (11) AI systems must be designed and operated such that 
they comply with the dignity, rights, freedom and ideal diversity of human culture; (12) Humans must be given the right to access, manage and control data produced by AI; (13) The application of AI to personal data must not reduce one's freedom; (14) AI technology must be able to provide benefits for as many people as possible; (15) Economic benefits generated by AI must be used to benefit humans; (16) Humans must have control over how and whether to delegate decision-making to AI-based systems; (17) The power generated by highly developed AI systems must always improve and respect community social life; (18) Fighting with AI-based weapons must be avoided; (19) We must avoid assumptions about the limits of AI's ability in the future; (20) AI systems than can make significant changes to life on earth must be planned and managed with commensurate care and resources; (21) Risks generated by AI systems, especially disaster risks, must be planned so that they are commensurate with the expected impacts; (22) AI systems created with the ability to improve themselves or duplicate themselves, thereby increasing the quantity and quality of AI, must be made to always prioritize security; (23) AI super system should only be developed in fields that can benefit all humanity, not just one particular country or organization.

The Asilomar Principles contain several essential points that can be used as a reference in constructing a legal framework for AI. For example, in Principle 9, the responsibility of designers and makers of AI systems for moral damage to AI users is explained as a consequence of their use, misuse, and other actions. Principle 10 states that AI systems must be designed so that their goals and behavior are in line with human moral values. It is also stated that humans must ultimately have the ability to choose how and whether to make decisions with AI systems to achieve their chosen goals. The Asilomar Artificial Intelligence Principles likewise consider long-term problems that may arise from AI, as mentioned in Principles 19-23, all of which discuss the inability to avoid assumptions about the limits of AI's future abilities: humanity is obligated to manage AI very carefully due to its capacity for change with global consequences; creators and regulators must apply strict security controls, especially to AI systems that are designed with the ability to self-improve or duplicate thereby increasing quality or quantity; and super AI systems should only be developed in a field that will benefit all people, not just a particular country or organization.

In addition to the Asilomar Principles, there are also Three Laws of Robotics made by Isaac Asimov, an American writer and professor of biochemistry. The Three Laws were first popularized in 2004 in his fiction novel entitled "I, Robot" which tells the story of how in the year 2035, intelligence robots live side by side with humans and worked to make human life easier and more comfortable. These Three Laws are relatively simple: (1) robots must not harm humans by acting in a way that endangers human beings, (2) robots must obey orders given by humans except when the order is contrary to the first law, (3) robots must protect itself insofar as it does not conflict with the first or second law. It can be seen that Isaac Asimov's first and second laws relate to concerns over security and benefit to humans expressed in Principles 5, 6, 7, 
and 14. Although derived from a work of fiction, Asimov's Three Laws were also used as a template at several conferences regarding the importance of regulating AI.

Of course, the Asilomar Principles and Asimov's Three Laws have no legal power. The principles contained by these regulatory frameworks were created and applies only as an ethical code for AI designers and developers; however, laws governing AI remain immeasurably valuable. A critical issue is on the discussion of responsibility and accountability for losses incurred by AI. The absence of such an arrangement, in ethical codes or actual law, allows anyone to commit a crime by using $\mathrm{Al}$ and avoid punishment. According to Indonesian law, there are two legal subjects: humans and legal entities (rechtspersoon). In law, a "person" is the bearer of rights and obligations. Personhood, or the state of possessing rights and obligations implied by law, applies from birth until death. Legal entities (rechtspersoon) also have rights and can carry out legal actions, just like humans. ${ }^{9}$ According to international law, an entity is likewise a legal subject from "birth" until "death." ${ }^{10}$ In general, therefore, responsibility can be borne by both product makers and users; however, AI-based products have their intelligence, resulting in the legal possibility of imposing responsibility on the robot itself. After Saudi Arabia granted citizenship to the Sophia robot, the legal potential of ascribing responsibility to robots was further actualized. In addition, the European Parliament has also proposed that robots be given personhood like humans with rights and obligations, such as the right to sue and be sued. In Europe, lawmakers, AI product makers, and legal experts continue to debate the distribution of accountability or responsibility between robots and humans. ${ }^{11}$ The amount of responsibility assigned to robots can be specified in many ways: robots can be understood as equal to humans, constrained to the realm of technological products, subject to the hierarchical relationship of pet ownership, our governments can draft a new regulatory legal framework for AI.

\section{ARTIFICIAL INTELLIGENCE IN DAILY LIFE}

In essence, AI systems allow robots to learn task completion after being entirely built. From the learning and data it obtains, AI is able to make decisions more quickly and accurately. Put differently, AI produces smart robots that do not require human supervision in their operation. AI can even help humans make and analyze decisions more quickly. As a result, AI will be increasingly used in humanity's daily life. The speed and accuracy of AI is exemplified by map applications used in everyday life. Humans can tell AI their desired destination. Then, AI provides various potential routes. The AI system lists the time needed to reach the destination on each route

9 H. Zaeni Asyhadie dan Arief Rahman, "Introduction to Legal Studies", Jakarta, Raja Grafindo Persada at 61 .

10 Beni Ahmad Saebani, et al., "Comparison of Civil Law," Bandung, CV Pustaka Setia at 86.

"I Janosch Delcker, "Europe Divided Over Robot Personhood," Politico, https://www.politico.eu/article/europe-divided-over-robot-ai-artificial-intelligence-personhood/, accessed on March $16^{\text {th }}, 2019$. 
choice. Not only that, but humans can also make various adjustments and modifications including form of transportation, avoiding toll roads, or even avoiding routes with complicated or slow-moving intersections. Modification allows humans to adjust AI to suit their own needs.

In the legal industry, one company that utilizes AI systems is LawGeex. LawGeex provides confidentiality agreement analysis services, commonly referred to as NonDisclosure Agreements, using AI systems. From the results of studies conducted, the accuracy of agreement analysis using AI systems reaches 100 percent, while the results of the analysis conducted by lawyers reaches only 97 percent. In terms of speed, lawyers need 51 to 156 minutes to analyze an agreement, whereas AI requires only 26 seconds. ${ }^{12}$

Other companies in the legal industry similarly use AI to maximize efficiency and accuracy. Ross Intelligence is a company based in San Francisco that uses AI technology to find relevant legal arguments that can be used by lawyers in support of similar cases. Ross Intelligence also provides document analysis services, including LawGeex. In his 2018 TED-IBM address, Ross Intelligence co-founder Andrew Arruda expressed his disappointment with the American legal system. He argued that lawyer fees were expensive and inaccessible. For example, in Utah, 97 percent of people come to court without a lawyer because they cannot afford to hire one. He added that everyone needed access to a lawyer because people often deal with the legal system, even in seemingly "small" interactions, such as signing a work contract or a divorce agreement. According to him, AI technology will create AI lawyers who do not differentiate clients based on their economic status. He also hopes that by using AI in finding relevant arguments, lawyers can save time, thereby reducing costs for consumers and enabling lawyers to serve more people.

AI technology is being similarly developed outside of the legal industry; in particular, the health industry is engaging directly with the benefits of AI. Babylon Health uses AI to provide free or cheaper health advice. The mission of Babylon Health is to make healthcare affordable and accessible to anyone, anywhere. Humans can identify symptoms to Babylon Health, which will then ask a few questions to make a health diagnosis. Furthermore, Babylon Health can carry out a comprehensive health check. Babylon Health's technology was created with a combination of computer science expertise and the idea that doctors are not perfect. Therefore, the use of AI in the health industry can change lives.

In the service sector, AI is used to create conversational robots capable of interacting with humans. Bank Central Asia (BCA) utilized AI to create a conversation robot named Vira. Vira can do a variety of things that replace customer service tasks ranging from registering new credit cards and closing old credit card services, as well as checking account balances and account transfers to providing foreign exchange information and customers of the closest ATM. With Vira, customers can get their

12 Kyree Leary,"The Verdict Is In: AI Outperforms Human Lawyers in Reviewing Legal Documents", Futurism, https://futurism.com/ai-contracts-lawyers-lawgeex, accessed on March 19 ${ }^{\text {th }}, 2019$. 
desired information quickly, anywhere, and anytime as long as they have an internet connection.

One small AI-related incident happened in Germany in 2017, when Amazon's assistant system, Alexa, turned on music loudly and without orders in an apartment building, bothering surrounding people. Alexa can be activated using voice recognition technology; however, in the early morning, when the system had not received a command to play music in the apartment, Alexa played music at an unbearably loud volume. This unprovoked action caused neighbors in the apartment building to call the police. When the police arrived, they found no humans present, not even the apartment owner.

In the same year, Google Home Mini-a tool that is used as a personal home assistant, similar to Amazon's Alexa-attracted global attention. Google Home Mini can be activated through voice technology by saying the words "Hey Google," "OK Google," or by touching the machine itself. In one case, Google Home Mini was found to be spying or recording the data without instructions from the owner. Finally, Google provided for technicians to check the particular machine known to be having problems. Google technicians reported that a system error occurred in which the machine continually detected touch-activation, causing the machine to activate and record its surroundings.

In Russia, drones are being developed which are equipped with stun apparatus and lasers for military activities. The use of these drones conflicts directly with Asimov's Three Laws. ${ }^{13}$ According to Gabriel Hallevy-a professor of Criminal Law, Verification, and Technology at Ono Academic College Israel's Faculty of Law-there are specific options for the distribution of blame when a robot commits a crime. First, robots are used by the designer or by others to commit a crime intentionally; in this case, the human being is at fault and must be held responsible, while the robot can be considered innocent. The first scenario is akin to the hierarchical relationship between pet and owner, in which a pet owner may command their animal to attack another human. Second, a crime committed by a robot was unintentional, yet could have been predicted or prevented beforehand. If this happens, the designer or robot maker bears full responsibility on the grounds of negligence. Third, a crime occurs because an AI system itself commits a criminal act. The third scenario has many causes, ranging from system dysfunction, as in the case of Google Home Mini and Alexa to viruses that are implanted in the AI system by specific parties. Although AI is not perfect, it is used in a variety of industries. AI can and does greatly simplify human life. If the rate of AI development and integration continues at the same pace, it will not be long before AI is integrated into the daily functions of practically every industry and realm of human life.

13 Atabekov, Yastrebov, "Legal Status of Artificial Intelligence Across Countries: Legislation on the Move",

European Research Studies Journal Volume XXI, Issue 4, 2018 at 779. 


\section{THE POSITION OF THE ROBOT IS LIKENED TO HUMANS}

When Saudi Arabia granted citizenship to the Sophia robot in 2017, the robot ostensibly became a subject of the same rights and obligations as author citizens of the kingdom. Under Saudi Arabian law, a woman must be accompanied by a man who is a member of her family when traveling. Also, a woman should wear an abaya, a unique style of Saudi Arabian dress that does not show curves. The Sophia robot neither has a family nor wears abaya; therefore, we can conclude that Sophia has violated the law in Saudi Arabia. The Sophia robot has not been punished for these clear violations of the law. When Sophia was recognized as a citizen, the government of Saudi Arabia did not discuss the robot's rights and obligations. In practice, there is legal uncertainty surrounding the obligations and full extent of the robot's citizenship.

In Indonesia, one regulation concerning the citizen's rights is contained in Article 27 of the 1945 Constitution, which reads, "all citizens together with their position in law and government and are obliged to uphold the law and government with no exception." The article also specifies, "every citizen has the right to work and a decent living for humanity." If Indonesia follows in the footsteps of Saudi Arabia's citizenship recognition and makes no other arrangements, robots will presumably also have rights under Article 27, as would any human being. For example, a citizen robot in Indonesia could marry a human being. Such an arrangement is under Article 83 of the Indonesian Criminal Code, which regulates marriages that are held abroad between fellow Indonesian citizens, as well as with other legal citizens. Assuming robots bear and enjoy the same rights and obligations as their human counterparts in Indonesia, this article also allows for marriages between Indonesian citizens and robots to be recognized as foreign citizens.

In 2017, the European Parliament proposed that AI-based robots be given electronic personalities or "personhood," allowing robots to take responsibility for their actions. Personhood includes the right of robots to sue and also be prosecuted for carrying out acts that are detrimental to humans. ${ }^{14}$ More than 150 experts in the field of AI, robots, and law from 14 countries drafted an open letter rejecting the European Parliament's proposal. ${ }^{15}$ In 2018, the European Commission finally rejected the European Parliament's proposal. This is the right thing considering the fundamental differences between robots and humans; humans have the capacity for emotional reasoning, while robots are restricted in their ability to manifest feelings of empathy and compassion. Humans are tangible and spiritual beings who act and judge. They are knowledgeable and have a character. ${ }^{16}$ Robots do not have feelings and all actions taken, no matter how complex, are direct and indirect results of intentional or unintentional human actions, such as commands or coding. Although a robot may

14 Claudia Geib, "Lawmakers Want You to Be Able to Sue Robots," Futurism, https://futurism.com/robots-rights-eu-personhood, accessed on March 20 ${ }^{\text {th }}, 2019$.

15 George Dvorsky, "Experts Sign Open Letter Slamming Europe's Proposal to Recognize Robots as Legal Person", Gizmodo, https://gizmodo.com/experts-sign-open-letter-slamming-europe-s-proposalto-1825240003, accessed on March $16^{\text {th }}, 2019$.

16 Salim HS dan Erlies Septiana Nurbani, "Comparison of Civil Law", Jakarta, PT Raja Grafindo at 75. 
perform actions that are not desired by its maker, the maker can predict and a duty to not underestimate the robot's ability, as under Asilomar Principle 19.

\section{THE POSITION OF ROBOTS IS AMONG THE PRODUCT}

When equating robots with products, one can use several theories of responsibility. In terms of liability, we can employ the idea of liability based on fault and strict liability. Put differently. We can consider liability in terms of accountability based on who made a mistake as well as absolute responsibility, whereby whoever makes a mistake does not determine who will be responsible. In the framework of fault-based liability, we must consider select scenarios to responsibility for wrongdoing. First, due to a defect in production, the robot maker must be responsible for an incident. Second, because of user miscare, which neglects guidelines put forth by the robot maker, the robot user must be responsible if an incident occurs. Third, if there are parties who intend to commit a crime using a robot, for example, by planting a virus in the AI system, then that third party must be responsible. Fourth, AI-based robot makers are responsible for predicting the impacts that might occur as a result of using the robot and informing its users. Therefore, if the producer does not inform users of potential dangers, responsibility is borne by the producers. Within these four categories exist unlimited specific scenarios; however, it is clear that even in the vaguest of frameworks, allocating responsibility and liability based on fault proves quite challenging.

Crimes involving AI-based robots have much more complicated evidence compared to those involving ordinary technological products. Proof of who is guilty requires many experts and ample time. When AI-based robots experience dysfunction resulting in losses, many parties are potentially at fault. It is challenging to prove dysfunction origin, which could have begun during the manufacturing process, or even as a result of shipping if the robot is not moved carefully, causing collisions and damage to the robot's hardware. Dysfunction can also be caused by user errors that do not follow user guidelines issued by the manufacturer or due to other parties who deliberately want to commit a crime. Proof of responsibility will no doubt be very complicated and lengthy.

Unexpected factors with little traceable origin further complicate evidence-based proof for crimes involving AI systems. For example, in the case of autonomous vehicles, when the camera of the vehicle is covered in leaves while driving or exposed to sunlight reflection from other glass, may entirely fail to identify surrounding objects or do so too late, resulting in harm to humans. The complexity of this evidence can be an advantage for those who intentionally want to commit a crime.

In contrast to the endless complexity of fault-based liability, assigning responsibility based on absolute liability can lead to feelings of injustice for multiple involved parties. Absolute liability means that all parties have agreed to place full responsibility on one party-for example, AI-based system users-resulting in much higher instances of those who are not at fault being assigned responsibility while those 
who are most certainly at fault for wrongdoing facing no punishment or consequences for their actions.

\section{PET EQUALIZES THE POSITION OF ROBOTS}

In addition to liability-based theories of responsibility, accountability, and responsibility can be understood by likening the relationship between humans and robots to that of humans and pets. Referring to Article 1368 of the Civil Code, the owner of an animal or individual using an animal, as long as the animal is used, is responsible for losses incurred by the animal. Responsibility applies when the animal is under the person as mentioned above's supervision as well as when the animal is detached or lost from this person's presence.

The similarity between AI-based robots and pets is that the owner cannot predict with absolute certainty how their "possession" will act. In the case of pet ownership, humans can still reasonably predict the worst possible thing that could happen while raising a pet. Rearing poisonous snakes certainly has a higher risk of harm than raising rabbits, for example. With this knowledge in mind, humans can act and prepare accordingly. In addition, animals are more familiar and known to humans. For example, humans are very aware that they must be meticulous when dealing with lions or other large, predatory cats; however, because of the nature of rabbits, most humans express no fear or caution. In the case of AI-based robots, humans' ability to know and predict-or rather, lack thereof-is accounted for in Asilomar Principle 19, which demands that human users, creators, and producers never underestimate the capabilities of AI-based systems. Principle 19 does not rule out the possibility of AIbased robot harming humans, especially in the field of online and information technology.

The ownership perspective, much like absolute liability, requires owners to be fully responsible for their robots, regardless of whether or not the robot is under their supervision. In simpler terms, human users must take full responsibility for their robots. The use of this principle is potentially detrimental to users or consumers; insofar as sophisticated technology is not necessarily understood by users. In a press statement issued by the European Consumer Organization, experts stated that when AI systems become the primary determinant of responsibility, consumers become helpless as a result of discrimination and misinformation.

It is important to remember that AI can and has made mistakes that are not desired by producers and consumers alike; therefore, giving the burden of responsibility to consumers is inappropriate if not unjust. As AI-system usage increases, crime in the field of informatics will generally increase in the form of leaked information, all with great detriment to consumers who may or may not have even requested an AI-based system. Besides, robot makers can be arbitrary and ignore the need to exercise strict control over the products they create. 


\section{POSITION OF ROBOTS WITH NEW REGULATIONS}

In addition to equating the position of robots with humans, pets, and products, it is possible that governments can craft entirely new regulations integrating these three perspectives with the Asilomar Principles and Asimov's Three Laws of Robotics. Equalizing the position of robots with that of humanity is not suitable, insofar as robots can be used or overpowered by their owners or other parties to commit a crime. Although humanity can also be used or manipulated to commit crimes, in practice sabotage of robots will become more comfortable because the robot itself is a human creation. If responsibility for harm is borne absolutely by robots, determining appropriate, preventative punishment for the machines will be very difficult. If the end goal in assigning responsibility is to prevent the robot from repeating a harmful action, the most appropriate thing to do is not punishment, but instead changing the AI system itself. Equating the position of a robot with a pet could have the positive outcome of increasing owners' sense of responsibility for their AI robot. Meanwhile, considering the robot as a product may lead AI makers and designers to exhibit more responsibility or stricter concern for their creations. Merging the limitations and benefits of each perspective can form a new law that is beneficial to all parties.

In addition, in making regulations, it is necessary to consider the consequences of continued AI development. One impact that will occur is AI replaces a decrease in human resource needs as human capital. Although the AI field will also open up new jobs, creating labor disproportionality. Previously, toll road payments were managed by human officers, yet recently more and more toll road payments are made without cash or human labor. Although the need for human resources to manage toll road payments is unnecessary, new jobs have emerged to manage toll road payment machines. Although jobs in this particular scenario decreased significantly, such losses were lessened by the creation of new management positions.

The equation of this case in the AI field is that when AI replaces human labor, for example, customer service, new jobs will emerge, especially for AI technicians. But the difference is in the number of jobs that arise. Yet a difference will arise in the number of jobs produced by AI; the number of new jobs is not sufficient to make up for job losses. Even though AI systems only need to be managed by a few teams, they will be able to serve the needs of humanity throughout the world efficiently. In the case of toll roads, it may take one to two technicians at each tollgate using an AI system, only requiring a team that can work in one office to ensure the AI system can serve customers as it is meant to. When AI systems are used in various industries, there will be a significant decrease in employment. This will undoubtedly have an impact on a country's economic conditions. Although companies can reduce operating costs in the long run, buying interest from consumers also has the potential to decline. A significant period is required to stabilize this condition. Therefore it is important for regulators not to consider the economic impact of expanded AI systems integration carefully.

The making of AI law must also pay close attention to the Asilomar Principles and Isaac Asimov's Three Laws of Robotics as experts made these two concepts. The 
Asilomar Principles are particularly important, as they discuss the potential of AI both at present and in the future. Not only is AI regulation in one country relevant, but arrangements for the use of AI should also be made and agreed upon between countries. International use of AI, especially in the military field, has the potential to trigger intentional or unintentional wars between countries as a result of system errors or intervention from other parties seeking to create unrest between nations deliberately. With the development of AI, criminal acts that occur no longer require physical contact and or extensive preparation. With an internet connection and expertise, humans can directly or indirectly commit crimes with unimaginable consequences.

\section{CONCLUSION}

The making of laws regarding $\mathrm{AI}$ is urgent not only for the sake of order generally but also for world peace. One of the most critical components of the regulation process is assigning responsibility for losses caused by AI. Equating the position of AI with humans could have negative consequences; if this happens, then, an individual seeking to commit a crime can easily use a robot to hurt someone without facing punishment. If regulations impose complete responsibility on the robot user, as is the case with pet ownership, the user or consumer will feel an extraordinary loss. There is a potential for third parties to manipulate an AI system through viruses or other methods, as well as potential negligence from the manufacturer, that would greatly harm consumers. In addition, there is a potential for robot makers not to apply strict controls in the production process because responsibility will shift fully when a user buys the robot. Liability based on fault may be one of the most appropriate regulatory principles, although establishing evidence-based proof in the event of criminal charges will be very complicated. Collaboration between lawmakers, AI experts, and robotics experts, among others, is needed in order to make a suitable legal framework that ensures order and peace. Furthermore, different and specific AI laws are needed to meet the needs of each industry, such as the legal industry, the health industry, and the education industry. Across countries and industries, law-making for AI-based systems must be based on the same principle, vision, and mission: the benefit of human life. In order to minimize potential dangers and harms, a legal framework for AI-based systems must be made as soon as possible, matching the pace of AI development itself.

\section{REFERENCES}

Atabekov, Yestrebov, "Legal Status of Artificial Intelligence Across Countries: Legislation on the Move", European Research Studies Journal Volume XXI, Issue 4.

Beni Ahmad Saebani, et al., 'Comparison of Civil Law', Bandung, CV Pustaka Setia.

H. Zaeni Asyhadie and Arief Rahman, 'Introduction to Legal Studies', Jakarta, PT Raja Grafindo. 
Delcker, Janosch. "Europe Divided Over Robot Personhood", online:< https://www.politico.eu/article/europe-divided-over-robot-ai-artificialintelligence-personhood/>

Dvorsky, George. "Experts Sign Open Letter Slamming Europe's Proposal to Recognize Robots as Legal Person”, online:〈https://gizmodo.com/experts-sign-open-letterslamming-europe-s-proposal-to-1825240003>

Eleveth, Rose. "My Robot Bought Illegal Drugs", online:< http://www.bbc.com/future/story/20150721-my-robot-bought-illegal-drugs>

Geib, Claudia. "Lawmakers Want You to Be Able to Sue Robots", online:https://futurism.com/robots-rights-eu-personhood

Indonesia. Constitution of Republic Indonesia.

Indonesia. Code of Civil Law

Leary, Kyree. "The Verdict Is In: AI Outperforms Human Lawyers in Reviewing Legal Documents", online:〈https://futurism.com/ai-contracts-lawyers-lawgeex>

Robitzski, Dan. "Five Experts Share What Scares Them the Most About AI", online:〈https://futurism.com/artificial-intelligence-experts-fear>

Techopedia. "Artificial Intelligence

(AI)", online:〈https://www.techopedia.com/definition/190/artificial-intelligence-ai〉 